
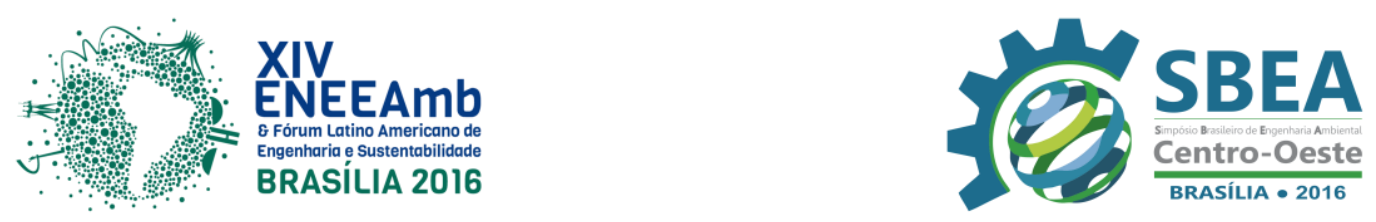

ENERGIAS RENOVÁVEIS

\title{
EFEITOS DA VARIAÇÃO DE pH E LUMINOSIDADE NO DESENVOLVIMENTO DA MICROALGA Scenedesmus sp VISANDO À PRODUÇÃO DE BIOCOMBUSTÍVEIS
}

Thiago Santos de Almeida Lopes - thiagosantos_al@outlook.com Universidade Estadual da Paraíba

André Luiz Alves da Silva - nadre.alas @ gmail.com

Universidade Estadual da Paraíba

Gustavo Ricardo Rodrigues Klein - gustavorrklein@ gmail.com

Universidade Estadual da Paraíba

Camila Bonfim Miranda - camilabonfimm@gmail.com

Universidade Estadual da Paraíba

Adriano Oliveira da Silva - adriano_able@ @otmail.com

Universidade Estadual da Paraíba

Weruska Brasileiro Ferreira - weruska.brasileiro@ pq.cnpq.br

Universidade Estadual da Paraíba

Resumo: O cultivo de microalgas tem se destacado como uma atraente alternativa para geração de bicombustíveis, devido à sua alta produtividade por unidade de área, uso de terras não aráveis e contribuir com a redução dos efeitos do superaquecimento global em razão do maior sequestro de $\mathrm{CO}_{2}$, que é emitido com a queima dos combustíveis fósseis. Várias espécies de microalgas também possuem a capacidade de sobreviver em ambientes extremos, adaptando o seu metabolismo de acordo com a alteração das condições ambientais. Desse modo, é importante analisar os parâmetros que influenciam em seu crescimento, como pH e luz, a fim de definir as condições que melhor favorecem seu desenvolvimento bem como buscar a otimização dos cultivos para uma maior produção de biomassa. Dentre as espécies de microalgas existentes, a Scenedesmus sp se destaca devido à sua elevada produtividade lipídica e de biomassa, o que potencializa a produção de biocombustíveis. Outro ponto que merece destacar é o beneficio ambiental promovido pelo cultivo de microalgas em especial a Scenedesmus sp em razão da mitigação das emissões de $\mathrm{CO}_{2}$ e possível biorremediação de águas residuárias. No presente estudo realizou-se cultivos da Scenedesmus sp em $\mathrm{pH}$ variando de 5,5 a 9, e fornecimento de luz variando de $6 \mathrm{~h}$ a $24 \mathrm{~h}$, onde verificou-se que a condição mais favorável para o cultivo da respectiva microalga visando à produção de biocombustíveis foi pH 9 e $12 \mathrm{~h}$ de luz, que resultaram numa velocidade específica máxima de crescimento de $0,0265 \mathrm{~h}^{-1}$, tempo de geração de $26,1509 \mathrm{~h}$ e produtividade de biomassa de $0,0757 \mathrm{~g} \cdot \mathrm{L}^{-1} \cdot \mathrm{d}^{-1}$. Estas condições indicam que a microalga Scenedesmus sp pode se adaptar facilmente em efluentes industriais e domésticos que apresentam alta alcalinidade e a não necessidade de iluminação artificial utilizando a própria energia solar em cultivos de grande escala. 
Palavras-chave: Microalgas, Biocombustíveis, Scenedesmus sp, Biorremediação

\section{INTRODUÇÃO E OBJETIVOS}

O cultivo de microalgas se mostra como uma opção em potencial para obtenção de biomassa visando à produção de biocombustíveis, devido à sua alta produtividade por unidade de área, uso de terras não aráveis e sequestro de $\mathrm{CO}_{2}$, além da capacidade de sobreviver em ambientes extremos, adaptando o seu metabolismo de acordo com a alteração das condições ambientais (CHIRANJEEVI \& MOHAN, 2016).

As microalgas do gênero Scenedesmus apresentam características fundamentais para a produção de biocombustíveis: são verdes, simples, com alta eficiência fotossintética, possuem a capacidade de acumular alto teor de amido e lipídios - valiosos para a produção do bioetanol e do biodiesel, respectivamente - e a sua estrutura celular simplifica o processamento dos biocombustíveis quando comparado com outras plantas complexas, podendo substituir os recursos agrícolas (CHNG et al., 2016).

De acordo com estudos taxonômicos e bioquímicos, o cultivo de microalgas pode ser fotoautotrófico, heterotrófico, mixotrófico e fotoheterotrófico (ANGELO et al., 2015). Dentre eles, o cultivo mixotrófico apresenta diversas vantagens, incluindo melhor redução de $\mathrm{CO}_{2}$, ausência de fotoinibição, maior crescimento celular e menor dificuldade para coleta de biomassa (CHANDRA et al., 2014). Nesse tipo de cultivo, as microalgas utilizam carbono orgânico e energia luminosa para o seu desenvolvimento.

A luz desempenha um papel central na produtividade de microalgas uma vez que fornece a energia necessária para as reações fotossintéticas, promovendo a conversão de nutrientes inorgânicos, dissolvidos no meio, em biomassa orgânica. A utilização de luz é, portanto, fundamental para o elevado rendimento da biomassa microalgal (SUTHERLAND et al., 2015).

$\mathrm{O}$ pH do meio de cultura é conhecido por ter grande influência no crescimento e na produção da biomassa de microalgas, uma vez que seu $\mathrm{pH}$ citosólico é neutro, ou ligeiramente alcalino, e suas enzimas celulares são sensíveis ao $\mathrm{pH}$ e podem se tornar inativas em condições ácidas (TRIPATHI et al., 2015). O pH está diretamente ligado com o bom funcionamento celular das microalgas, por isso o seu controle é de vital importância para o melhor desenvolvimento dos cultivos.

Então, sabendo que as condições ambientais estão diretamente relacionadas com o crescimento celular, a produtividade de biomassa e o acúmulo de lipídios, este estudo analisou a influência da luminosidade e do $\mathrm{pH}$ no crescimento da microalga Scenedesmus sp em cultivo autotrófico, buscando otimizar o seu desenvolvimento com vistas à produção de biocombustíveis.

\section{METODOLOGIA}

\subsection{Microalga e aclimatação dos cultivos}

O presente estudo foi realizado no Laboratório de Saneamento Ambiental do Centro de Ciências e Tecnologia da Universidade Estadual da Paraíba utilizando cepas da microalga de espécie Scenedesmus sp fornecidas pelo laboratório da Universidade Federal Fluminense.

A aclimatação dos cultivos foi realizada de modo a estabelecer um ambiente propício para a otimização do crescimento da Scenedesmus sp. As culturas foram desenvolvidas em Erlenmeyres, que foram utilizados como fotobioreatores e mantidos a temperatura de $26^{\circ} \mathrm{C} \pm 2$, com agitação de ar comprimido para garantir a homogeneização do meio, e expostos por um período de 12 horas à iluminação de 4.000 lux, proveniente de lâmpadas fluorescentes de $40 \mathrm{~W}$.

A Figura 1 representa a fotomicrografia das cepas da microalga Scenedesmus sp e as condições de aclimatação adotadas para o desenvolvimento das microalgas. 


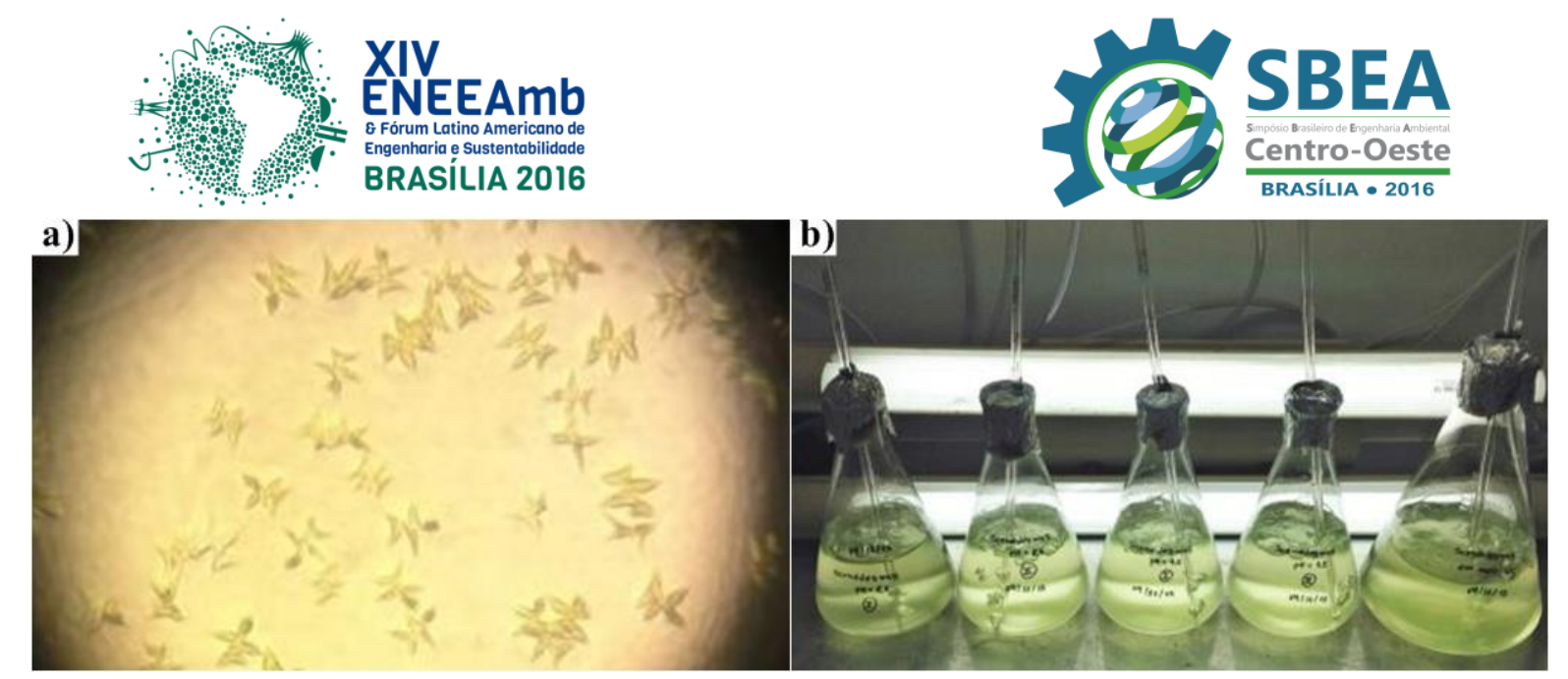

Figura 1 - a) Fotomicrografia da microalga Scenedesmus sp; b) Aclimatação dos cultivos

\subsection{Estratégia de estudo}

Para o cultivo da Scenedesmus sp foi utilizado o meio de cultura Wright's Cryptophyte (WC), desenvolvido por Guillard \& Lorenzen (1972), sob diferentes condições de pH e luminosidade, onde buscou-se analisar a influência de tais fatores no desenvolvimento da espécie em estudo.

As Tabelas 1 e 2 apresentam as condições de $\mathrm{pH}$ e luminosidade utilizados durante o presente estudo. Inicialmente, desenvolveram-se cultivos de Scenedesmus sp em meio de cultura WC com variadas alterações em seu $\mathrm{pH}$, mantendo-se as condições originais de aclimatação (12h de luminosidade). Em seguida, desenvolveram-se cultivos com variação no tempo de fornecimento de luz, utilizando-se o $\mathrm{pH}$ que proporcionou os melhores resultados de crescimento da respectiva microalga. Cada experimento teve duração total de 9 dias.

Tabela 1 - Condições de $\mathrm{pH}$ utilizadas nos cultivos da microalga Scenedesmus sp

\begin{tabular}{|c|c|c|c|}
\hline \multicolumn{3}{|c|}{ Valores de pH conferidos ao meio de cultura WC para o cultivo da Scenedesmus $\mathbf{s p}$} \\
\hline 5,5 & 6,5 & 7,5 & $\begin{array}{c}9,0 \text { (meio WC sem } \\
\text { alteração) }\end{array}$ \\
\hline
\end{tabular}

Tabela 2 - Condições de luminosidade utilizadas nos cultivos da Scenedesmus sp

Quantidade de horas de luz fornecidas por dia aos cultivos de Scenedesmus sp em meio WC

\begin{tabular}{l|c|c|c}
6,0 & 12,0 & 18,0 & 24,0
\end{tabular}

\subsection{Avaliação do crescimento}

Com o auxílio da microscopia óptica foi possível determinar o crescimento das microalgas avaliando-se a densidade celular em função do tempo de cultivo em cada uma das unidades experimentais. As amostras foram retiradas ao início dos cultivos e depois a cada $24 \mathrm{~h}$, para contagem de células em câmara de Neubauer, determinando, desse modo, a densidade celular expressa em número de células por mililitro de cultivo (células. $\mathrm{mL}^{-1}$ ).

A contagem de células foi realizada em triplicata. $\mathrm{O}$ número de células corresponde à média geométrica das três contagens. O tempo de cultivo foi expresso pela quantidade de dias decorridos desde o início da inoculação (período de adaptação - fase lag) até o alcance máximo da densidade celular (fase estacionária). Os gráficos de dispersão foram plotados para representar as curvas de crescimento da Scenedesmus sp, onde o eixo das abscissas corresponde ao o tempo de cultivo em dias e o eixo das ordenadas ao número de células. $\mathrm{mL}^{-1}$.

\subsection{Avaliação da cinética de crescimento algal}

A velocidade de crescimento é diretamente proporcional à concentração de microrganismos em um dado instante. A fração pela qual a população cresce na unidade de tempo é 

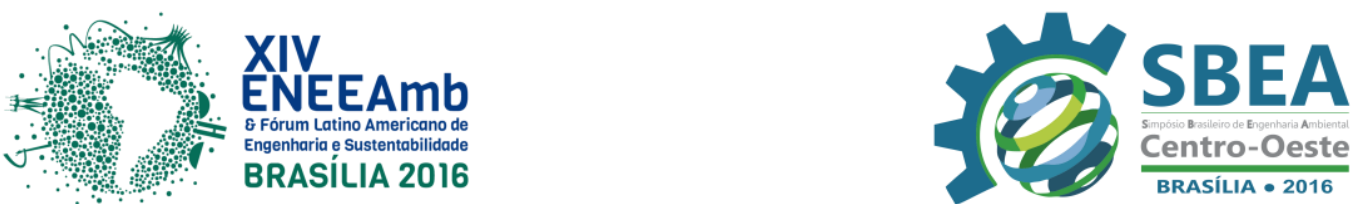

dada por $\mu_{\text {máx }}$, que representa a velocidade específica de crescimento e tem unidade de tempo $\mathrm{h}^{-1}$. Na fase exponencial (ou logarítmica) a velocidade específica de crescimento é constante e máxima, sendo $\mu_{\mathrm{x}}$ igual a $\mu_{\text {máx. }}$ A velocidade de crescimento foi calculada através da Equação (1).

$$
\ln (\mathrm{x})=\mu_{\text {máx }} \cdot\left(\mathrm{t}-\mathrm{t}_{\mathrm{i}}\right)+\ln \left(\mathrm{x}_{\mathrm{i}}\right)
$$

A representação de $\ln (\mathrm{x})$ versus o tempo de cultivo, na fase exponencial, resulta em uma reta com coeficiente angular igual à velocidade específica máxima de crescimento $\mu_{\text {máx. A fase }}$ exponencial também é caracterizada pelo tempo de geração $\left(\mathrm{t}_{\mathrm{g}}\right)$, que é o tempo necessário para dobrar o valor da concentração celular $\left(\mathrm{x}=2 \mathrm{x}_{\mathrm{i}}\right)$. $\mathrm{O}$ tempo de geração foi calculado através da Equação (2).

$$
\mathrm{t}_{\mathrm{g}}=\ln (2) / \mu_{\text {máx }}=0,693 / \mu_{\text {máx }}
$$

\subsection{Avaliação da produtividade de biomassa}

As análises de produtividade da biomassa cultivada em laboratório foram realizadas em triplicata no início e fim dos cultivos. Foram utilizadas membranas de acetato de celulose de porosidade de $0,45 \mu \mathrm{m}$, apresentadas na Figura 2, para realizar filtração a vácuo das amostras contendo biomassa de Scenedesmus sp, realizando-se a secagem em estufa na temperatura de $60^{\circ} \mathrm{C}$ até atingir peso constante e posterior pesagem da biomassa seca.

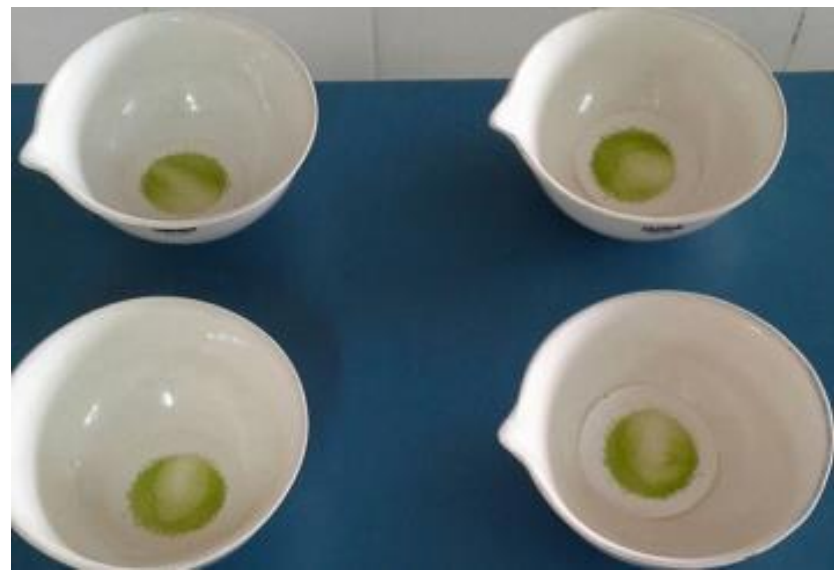

Figura 2 - Membranas de acetato de celulose com biomassa da microalga Scenedesmus sp

A produtividade foi determinada por meio da Equação (3), onde a biomassa seca inicial corresponde à quantidade de biomassa no início do cultivo e a biomassa seca final corresponde à quantidade de biomassa ao término do cultivo.

$$
\text { Produtividade }=\left(\text { biomassa }_{\text {seca,final }}-\text { biomassa }_{\text {seca,inicial }}\right) /\left(\text { tempo } \text { final }_{\text {final }}-\text { tempo }_{\text {inicial }}\right)
$$

\section{RESULTADOS E DISCUSSÕES}

\subsection{Scenedesmus sp sob diferentes condições de pH}

A fim de verificar a influência que a variação de $\mathrm{pH}$ exerceu sobre o crescimento da microalga Scenedesmus sp, foram elaborados cultivos variando-se o $\mathrm{pH}$ do meio de cultura WC numa faixa de 5,5 a 9,0, mantendo-se as aclimatações físicas estabelecidas inicialmente. A Figura 3 ilustra as curvas de crescimento da respectiva microalga sob diversas condições de $\mathrm{pH}$, durante o tempo de cultivo 9 dias. Todos os cultivos iniciaram com uma concentração de células na ordem de $10^{5}$ e, apesar da variação de $\mathrm{pH}$, alcançaram quantidades semelhantes de número de células. 

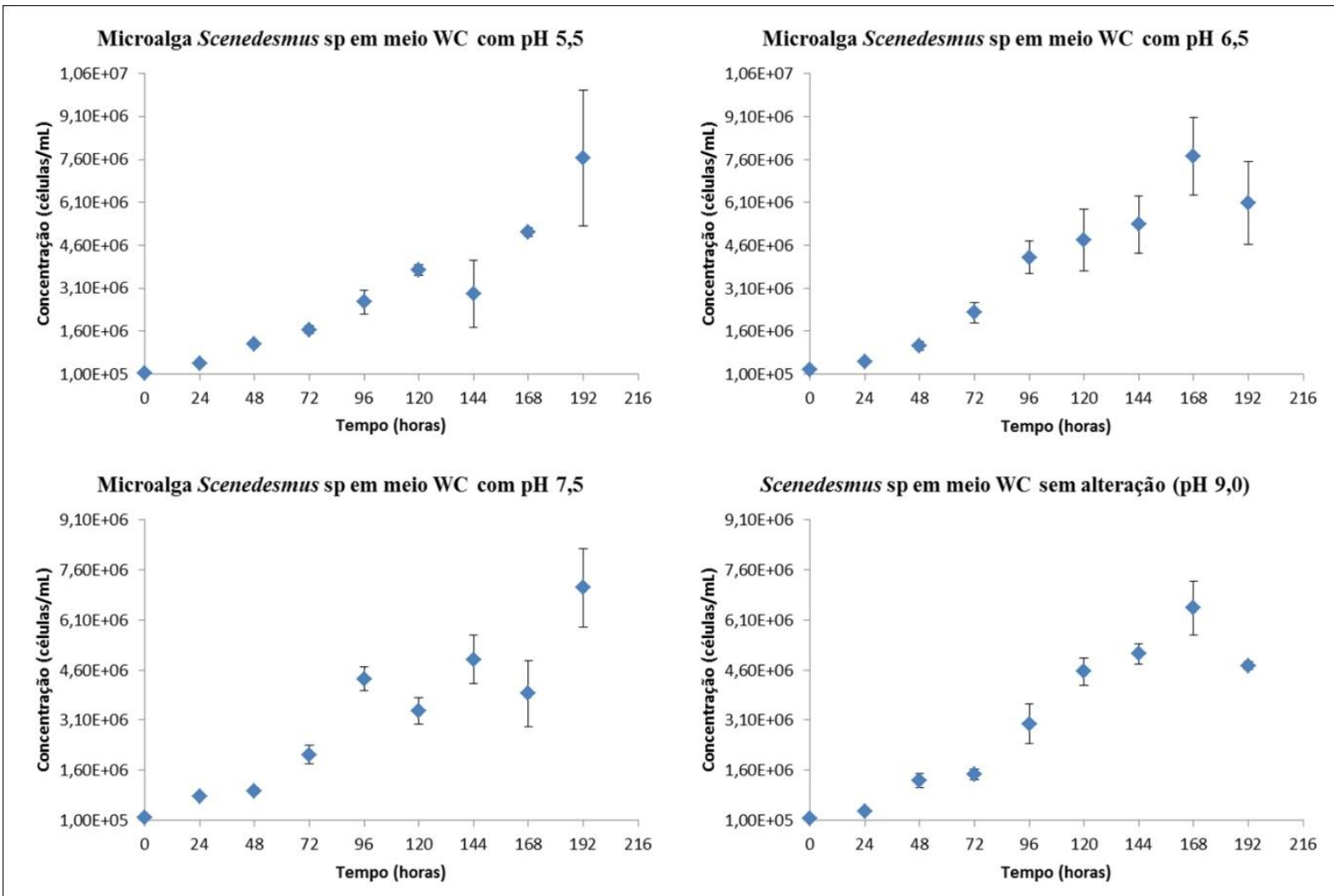

Figura 3 - Curvas de crescimento da Scenedesmus sp sob diversas condições de $\mathrm{pH}$

O cultivo de Scenedesmus sp em meio WC com pH 6,5 foi o que desenvolveu o maior número de células, assim como os melhores resultados de velocidade específica máxima de crescimento e tempo de geração, apresentados na Tabela 3. Por outro lado, desenvolveu a menor produtividade de biomassa entre os demais. Comparando esse cultivo com o de $\mathrm{pH}$ 9,0 (meio WC sem alteração), verificou-se que há pouca diferença entre os valores de número máximo de células alcançado, velocidade específica máxima de crescimento e tempo de geração, utilizando-se como fator decisivo da melhor condição a produtividade em biomassa, que é essencial para a produção de biocombustíveis. Desse modo, observando-se que o cultivo onde não houve alteração de $\mathrm{pH}$ proporcionou a melhor produtividade em biomassa, selecionou-se essa condição química para os demais experimentos, ou seja, deu-se continuidade aos estudos com Scenedesmus sp sem alterar o $\mathrm{pH}$ do meio de cultura WC.

Tabela 3 - Velocidades específicas máximas, tempos de geração e produtividades de biomassa dos cultivos de Scenedesmus sp em meio WC sob diversas condições de $\mathrm{pH}$

\begin{tabular}{|c|c|c|c|c|}
\hline pH do meio de cultura & $\begin{array}{c}\text { Velocidade } \\
\text { específica } \boldsymbol{\mu}_{\text {máx }}\left(\mathbf{h}^{\mathbf{- 1}}\right)\end{array}$ & $\mathbf{R}^{\mathbf{2}}$ & $\begin{array}{c}\text { Tempo de } \\
\text { geração }(\mathbf{h})\end{array}$ & $\begin{array}{c}\text { Produtividade de } \\
\text { biomassa }\left(\mathbf{g . L}^{-\mathbf{1}} \cdot \mathbf{d}^{-\mathbf{1}}\right)\end{array}$ \\
\hline 5,5 & 0,0203 & 0,9930 & 34,1380 & 0,0550 \\
\hline $\mathbf{6 , 5}$ & $\mathbf{0 , 0 2 8 5}$ & 0,9990 & $\mathbf{2 4 , 3 1 5 8}$ & $\mathbf{0 , 0 4 7 0}$ \\
\hline 7,5 & 0,0242 & 0,9503 & 28,6364 & 0,0633 \\
\hline $\begin{array}{c}\mathbf{9 , 0} \text { (sem alteração do } \\
\text { meio de cultura) }\end{array}$ & $\mathbf{0 , 0 2 6 5}$ & 0,9086 & $\mathbf{2 6 , 1 5 0 9}$ & $\mathbf{0 , 0 7 5 7}$ \\
\hline
\end{tabular}

Os valores elevados do coeficiente de determinação $\left(R^{2}\right)$, também expressos na Tabela 3 , indicam que houve um excelente ajuste amostral ao modelo de estudo utilizado. 

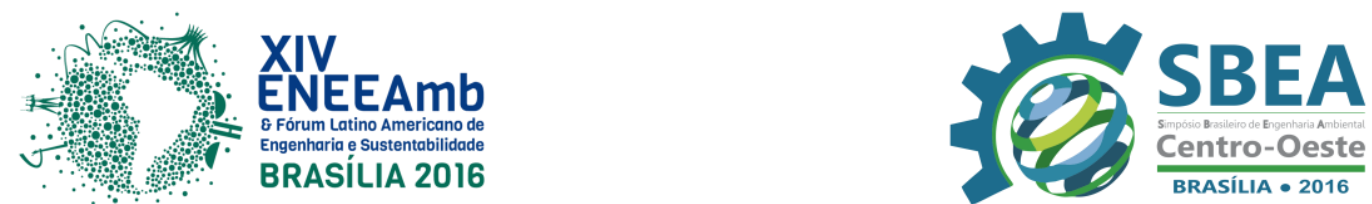

Tripathi et al. (2015) cultivaram Scenedesmus sp em meio de cultura BG-11, com pH 7,4 e $18 \mathrm{~h}$ de luz, e analisou a influência do $\mathrm{pH}$ alterando-o numa faixa entre 7 e 10, onde a microalga obteve sua maior produtividade em $\mathrm{pH} 8$, mostrando que seu meio de cultivo deve ser alcalino. Chiranjeevi e Mohan (2016) também verificaram maior produção de biomassa em pH alcalino $(8,5)$, que em pH ácido (6) e neutro $(7,5)$. Fato reforçado no presente estudo, onde a produtividade da respectiva microalga aumentou juntamente com o $\mathrm{pH}$.

Aburai et al. (2015) cultivaram a Scenedesmus sp em Bold's Basal Medium (meio de cultura BBM) autoclavado, com pH ajustado para 8, em Erlenmeyers de $500 \mathrm{~mL}$, e obtiveram uma velocidade específica máxima de crescimento de $0,0112 \mathrm{~h}^{-1}$. Comparando-se esse dado com o presente estudo, verifica-se que o meio $\mathrm{WC}$ em $\mathrm{pH}$ alcalino, além de favorecer a produção de biomassa, também favoreceu a cinética de crescimento da microalga, cuja velocidade específica máxima de crescimento alcançou o valor de $0,0265 \mathrm{~h}^{-1} \mathrm{em} \mathrm{pH} 9$.

Sutherland et al. (2015) investigaram os efeitos da adição de $\mathrm{CO}_{2}$ no cultivo de microalgas em águas residuárias, ao longo de um gradiente de $\mathrm{pH}$, e observaram melhor eficiência fotossintética quando o $\mathrm{pH}$ das culturas foram reduzidos para 8 e 6,5, onde o $\mathrm{pH}$ 6,5 promoveu o melhor rendimento de biomassa das microalgas Micractinium bornhemiense e Pediastrum boryanum, porém reduziu de forma significativa a eficiência na absorção de nutrientes. Isso mostra que, para o tratamento de águas residuárias, o pH alcalino favorece a remoção de nutrientes, de modo que a microalga Scenedesmus sp, ao desenvolver melhor produtividade em $\mathrm{pH} \mathrm{9,} \mathrm{é} \mathrm{uma} \mathrm{forte} \mathrm{candidata} \mathrm{para} \mathrm{remediação} \mathrm{de} \mathrm{efluentes}$ alcalinos.

\subsection{Scenedesmus sp sob diferentes condições de luminosidade}

A Figura 4 ilustra as curvas de crescimento da microalga Scenedesmus sp em $6 \mathrm{~h}, 18 \mathrm{~h}$ e 24h de luz, juntamente com a do cultivo de $12 \mathrm{~h}$ de luz, já apresentada na Figura 3 ( $\mathrm{pH} \mathrm{9,0).} \mathrm{Os}$ cultivos foram realizados mantendo-se a melhor condição de $\mathrm{pH}$ definida na primeira parte do estudo.

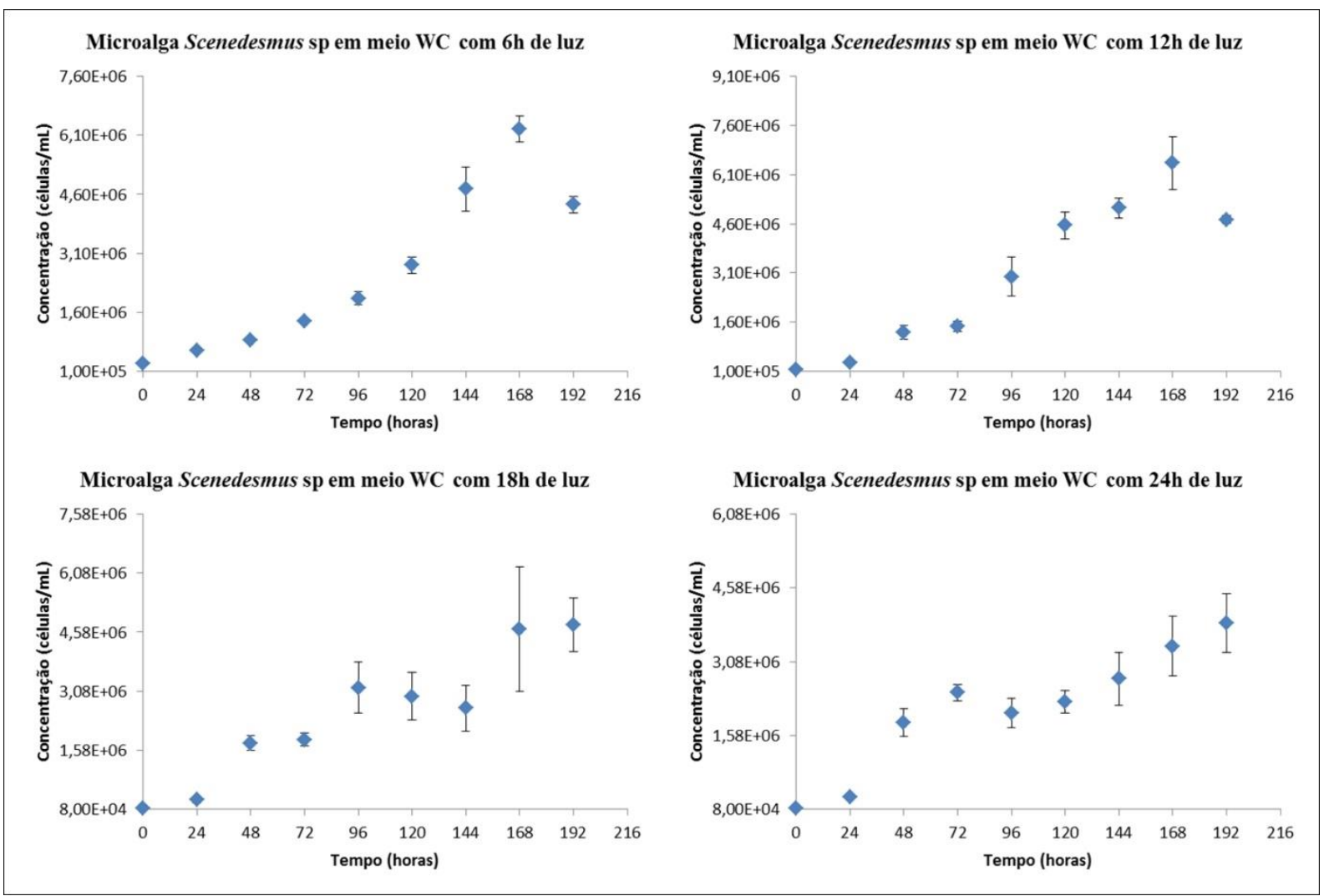

Figura 4 - Curvas de crescimento da microalga Scenedesmus sp em meio WC (sem alteração de $\mathrm{pH}$ ) com variação na disponibilidade de luz 

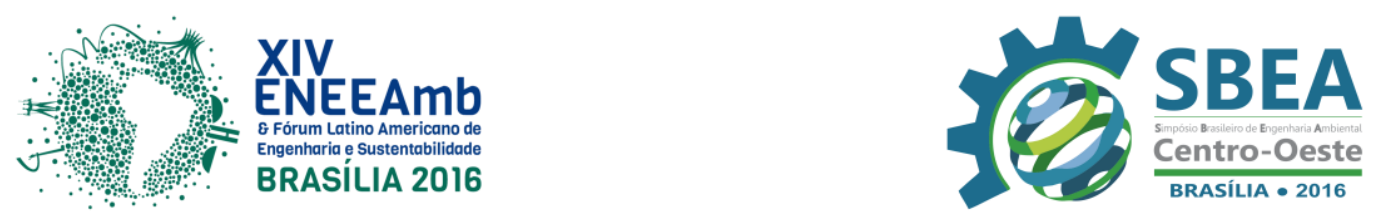

Os cultivos com $6 \mathrm{~h}$ e $12 \mathrm{~h}$ de luz iniciaram com um número de células na ordem de $10^{5}$, enquanto que os com $18 \mathrm{~h}$ e $24 \mathrm{~h}$ de luz iniciaram com $10^{4}$. Por esse motivo, os dois primeiros alcançaram maiores valores de número de células que os demais. No entanto, verifica-se que os cultivos com menor luminosidade apresentaram uma maior fase lag, o que corresponde a um lento crescimento nos primeiros dias após o inóculo. Já os cultivos com maior disponibilidade de luz rapidamente chegaram à fase exponencial, apresentando acelerado crescimento já no segundo dia após o inóculo.

Para uma melhor análise da influência da radiação luminosa sobre a microalga Scenedesmus sp, a Tabela 4 apresenta os dados de velocidade específica máxima de crescimento, tempo de geração e produtividade de biomassa dos cultivos com 6h, 12h, 18h e $24 \mathrm{~h}$ de iluminação.

Observa-se que quanto maior a disponibilidade de luz, maior serão a velocidade específica máxima de crescimento e a produtividade de biomassa, assim como menor será o tempo de geração. Fato comprovado em estudos realizados por Chiranjeevi e Mohan (2016), que mostraram uma maior produtividade de biomassa de microalgas na presença de luz que na ausência.

Han et al. (2015) estudaram a influência da luz no crescimento da Chlorella sp, fornecendo energia com intensidade na faixa de 2.000-10.000 lux, e observaram que a taxa de crescimento e o rendimento de biomassa seca melhoravam à medida que se aumentava a intensidade luminosa, porém, 6.000 lux satisfez completamente a necessidade das microalgas. No atual estudo, onde se forneceu energia com intensidade de $4.000 \mathrm{lux}$, verificou-se que o maior tempo de luminosidade ( $24 \mathrm{~h}$ de luz por dia) favoreceu o melhor desenvolvimento da microalga, porém, o tempo de fornecimento de luz de $12 \mathrm{~h}$ foi suficiente para as necessidades microalgais. Além disso, em larga escala se pretende simular as condições externas do meio ambiente, fato da indicação do tempo de luminosidade de $12 \mathrm{~h}$ para o cultivo de Scenedesmus sp.

Tabela 4 - Velocidades específicas máximas, tempos de geração e produtividades de biomassa dos cultivos de Scenedesmus sp sob diversas condições de luminosidade

\begin{tabular}{|c|c|c|c|c|}
\hline $\begin{array}{c}\text { Horas de luz } \\
\text { por dia }\end{array}$ & $\begin{array}{c}\text { Velocidade específica } \\
\boldsymbol{\mu}_{\text {máx }}\left(\mathbf{h}^{-\mathbf{1}}\right)\end{array}$ & $\mathbf{R}^{\mathbf{2}}$ & $\begin{array}{c}\text { Tempo de } \\
\text { geração (h) }\end{array}$ & $\begin{array}{c}\text { Produtividade de } \\
\text { biomassa }\left(\mathbf{g . L}^{-\mathbf{1}} . \mathbf{d}^{-\mathbf{1}}\right)\end{array}$ \\
\hline 6,0 & 0,0161 & 0,9986 & 43,0435 & 0,0464 \\
\hline 12,0 & 0,0265 & 0,9086 & 26,1509 & 0,0757 \\
\hline $\mathbf{1 8 , 0}$ & $\mathbf{0 , 0 2 9 1}$ & 0,8056 & $\mathbf{2 3 , 8 1 4 4}$ & $\mathbf{0 , 0 8 4 6}$ \\
\hline $\mathbf{2 4 , 0}$ & $\mathbf{0 , 0 4 2 0}$ & 0,8556 & $\mathbf{1 6 , 5 0 0 0}$ & $\mathbf{0 , 1 1 6 5}$ \\
\hline
\end{tabular}

\section{CONSIDERAÇÕES FINAIS}

Através do estudo da microalga Scenedesmus sp sob diversas condições de $\mathrm{pH}$ e tempos de iluminação, visando a produção de biocombustíveis, pôde-se concluir:

- Valores de $\mathrm{pH}$ do meio de cultura WC entre a faixa 5,5-9,0 proporcionaram um bom desenvolvimento da microalga Scenedesmus sp, que alcançou a produtividade de biomassa máxima de 0,0757 g.L $\mathrm{L}^{-1} \cdot \mathrm{d}^{-1}$, em meio WC sem alteração química (pH 9,0).

- A disponibilidade de luz se apresentou como fator essencial para o desenvolvimento da microalga em estudo, mostrando que quanto maior o tempo de luminosidade, maior a velocidade específica máxima de crescimento e a produtividade de biomassa, e menor o tempo de geração, sendo o tempo de $12 \mathrm{~h}$ de luz definido como condição ideal para o cultivo em larga escala, possibilitando o aproveitamento da energia solar e dispensando a iluminação artificial. 

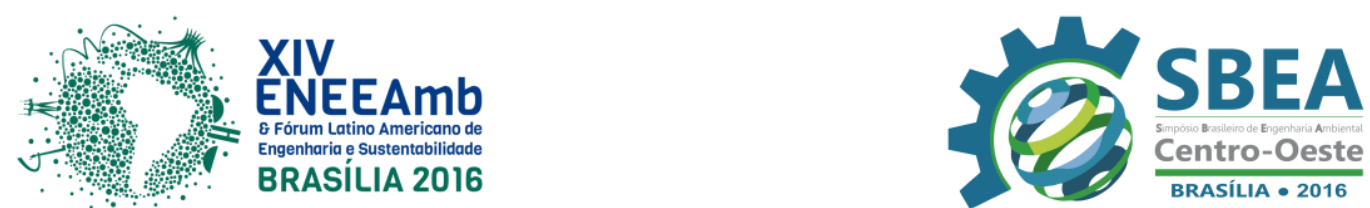

Agradecimentos

Ao CNPq pelo financiamento do projeto.

\section{REFERÊNCIAS}

ABURAI, Nobuhiro; SUMIDA, Daichi; ABE, Katsuya. Effect of light level and salinity on the composition and accumulation of free and ester-type carotenoids in the aerial microalga Scenedesmus sp.(Chlorophyceae). Algal Research, v. 8, p. 30-36, 2015.

ANGELO, Elisangela Andrade; ANDRADE, Diva Souza; COLOZZI FILHO, Arnaldo. Cultivo nãofotoautotrófico de microalgas: uma visão geral. Semina: Ciências Biológicas e da Saúde, v. 35, n. 2, p. 125-136, 2015.CHANDRA, Rashmi et al. Regulatory function of organic carbon supplementation on biodiesel production during growth and nutrient stress phases of mixotrophic microalgae cultivation. Bioresource technology, v. 165, p. 279-287, 2014.

CHIRANJEEVI, P.; MOHAN, S. Venkata. Critical parametric influence on microalgae cultivation towards maximizing biomass growth with simultaneous lipid productivity. Renewable Energy, 2016.

CHNG, Lee Muei; CHAN, Derek JC; LEE, Keat Teong. Sustainable production of bioethanol using lipid-extracted biomass from Scenedesmus dimorphus.Journal of Cleaner Production, 2016.

GUILLARD, Robert RL; LORENZEN, Carl J. YELLOW-GREEN ALGAE WITH CHLOROPHYLLIDE C1, 2. Journal of Phycology, v. 8, n. 1, p. 10-14, 1972.

HAN, Fei et al. Optimization and lipid production enhancement of microalgae culture by efficiently changing the conditions along with the growth-state. Energy Conversion and Management, v. 90, p. 315-322, 2015.

SUTHERLAND, Donna L. et al. The effects of $\mathrm{CO}_{2}$ addition along a $\mathrm{pH}$ gradient on wastewater microalgal photo-physiology, biomass production and nutrient removal. Water research, v. 70, p. 9$26,2015$.

TRIPATHI, Ritu; SINGH, Jyoti; THAKUR, Indu Shekhar. Characterization of microalga Scenedesmus sp. ISTGA1 for potential $\mathrm{CO}_{2}$ sequestration and biodiesel production. Renewable Energy, v. 74, p. 774-781, 2015. 